\title{
Establishment and characterization of breast cancer organoids from a patient with mammary Paget's disease
}

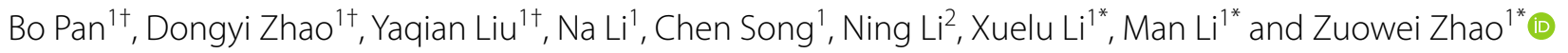

\begin{abstract}
Background: Mammary Paget's disease (MPD) is an uncommon cutaneous intraepithelial malignancy with ulceration of the nipple or areola. Its pathogenesis and genomic mutation remain largely unknown and no cell lines are established from primary tumors.

Methods: We collected surgical tumor specimens from a 65-year-old Chinese woman diagnosed with MPD and established patient-derived breast cancer (BC) organoids from MPD using organoid culture technology.

Results: We successfully propagated BC organoids from a patient with MPD for more than 6 months. The organoids were cultured for long-term expansion without any change in spherical organoid morphology. Besides, the spherical organoid morphology did not change when they underwent cryopreservation after resuscitation. The H\&E staining and immunohistochemistry analyses showed the similar morphological and histological features of the organoids compared with their paired original BC tissues. The organoids retained positive expression of breast cancer biomarkers: estrogen receptor, progesterone receptor, antigen Ki-67 and negative expression of human epidermal growth factor receptor 2 . We also showed that MPD organoids recapitulated the unique genomic landscape including copy number alterations, mutational load, mutational signatures and cancer gene mutations by whole genome sequencing. In situ senescence-associated acid beta galactosidase assay confirmed senescence phenomenon existed in the process of organoids culture and there was no significant difference in the proportion of senescent organoids after organoid passage and resuscitation.
\end{abstract}

Conclusions: Our results suggested that an effective platform for ex vivo BC organoids from MPD patients could be used to explore clinicopathological and genomic characteristics of these patients.

Keywords: Breast cancer, Paget's disease, Organoid culture, Genome sequencing

\section{Background}

Paget's disease (PD) is an uncommon cutaneous intraepithelial malignancy characterized histopathologically by large epidermal adenocarcinoma cells (Paget's cells)

\footnotetext{
*Correspondence: dmulixuelu@163.com; dmuliman@163.com; dmuzhaozuowei@163.com

${ }^{\dagger}$ Bo Pan, Dongyi Zhao and Yaqian Liu contributed equally to this work ${ }^{1}$ Department of Oncology \& Department of Breast Surgery, The Second Hospital of Dalian Medical University, Dalian 116023, China

Full list of author information is available at the end of the article
}

containing abundant mucin [1]. According to the affected anatomic locations, PD is classified as mammary Paget's disease (MPD) and extramammary Paget's disease (EMPD). MPD is characterized by the eczematous eruption and ulceration of the nipple or areola [2]. The MPD incidence of all breast cancer (BC) is approximately $1 \%$, and more than $90 \%$ of Paget's disease of the nipple is associated with underlying intraductal carcinoma (DCIS) or infiltrating duct carcinoma (IDC) [1,3]. Recent studies have confirmed that MPD conjunction with invasive cancer had worse prognosis [4]. Based on the overall

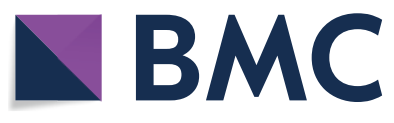

(c) The Author(s) 2020. This article is licensed under a Creative Commons Attribution 4.0 International License, which permits use, sharing, adaptation, distribution and reproduction in any medium or format, as long as you give appropriate credit to the original author(s) and the source, provide a link to the Creative Commons licence, and indicate if changes were made. The images or other third party material in this article are included in the article's Creative Commons licence, unless indicated otherwise in a credit line to the material. If material is not included in the article's Creative Commons licence and your intended use is not permitted by statutory regulation or exceeds the permitted use, you will need to obtain permission directly from the copyright holder. To view a copy of this licence, visit http://creativeco mmons.org/licenses/by/4.0/. The Creative Commons Public Domain Dedication waiver (http://creativecommons.org/publicdomain/ zero/1.0/) applies to the data made available in this article, unless otherwise stated in a credit line to the data. 
survival, MPD patients with PD-IDC had worse prognosis (5-year survival rate $=84.1 \%)$ compared with MPD patients with PD-DCIS (5-year survival rate $=97.5 \%$ ) [5]. The pathogenesis of PD has implications for the optimal treatment. The exact pathogenesis of the carcinoma of the epidermis is poorly understood, and debated as to whether it arises from the underlying breast parenchyma or the epidermal cells. There are two main theories proposed including the epidermotropic theory and the intraepidermal origin theory [6]. Epidermotropic theory declares that Paget's cells originate from underlying carcinoma cells that migrate into the epidermis, whereas the intraepidermal origin theory states that Paget's cells are the result of in situ oncogenic changes in epidermal cells of the apocrine gland ducts or pluripotent keratinocyte stem cells [6].

Recently, Zhang et al. [7] performed whole-exome sequencing on 41 pairs of PD tumor and normal skin samples to reveal the molecular landscapes of PD. They found that MPD and EMPD had the similar genomic aberrations, especially in genes involved in chromatin remodeling processes, such as KMT2C and ARID2. Further, MPD and underlying breast ductal carcinomas are likely independent oncogenic events. Gatalica et al. [8] investigated the molecular differences between MPD and EMPD, and found that PIK3CA and TP53 mutations were most common in MPD. Although rare, several other genomic alterations were also detected in MPD. However, the full genomic mutational landscapes of MPD remain uncharacterized and genomics-related research is still scarce. For less frequently occurring $\mathrm{BC}$ or special pathological types, due to the lack of a corresponding pre-clinical cell culture model, it is difficult to study the tumorigenesis, the phenotypic and genetic heterogeneity of this type of $\mathrm{BC}$ which hampers therapeutic innovation. We need to develop a viable and reliable method to improve the therapeutic effects of $\mathrm{BC}$ patients with $\mathrm{PD}$.

Three-dimensional (3D) organoid culture models open opportunities for both fundamental and translational cancer research. The organoids can be grown from primary patient material of a wide range of tumor tissues, such as kidney [9], colorectal [10], pancreas [11], lung [12] and breast cancer [13]. Tumor-derived organoids recapitulate and maintain the genetic heterogeneity of native tumor tissue over time, and have predictive value for individual patient drug responses [9, 10]. We previously performed two-dimensional (2D) culture technology to culture tumor cells from endometrial cancer patients [14] and breast cancer patients with leptomeningeal metastasis [15]. However, the tumor cells gradually underwent senescence after six or seven passages and could not expand as long as cancer cell lines. Afterwards, we successfully established a BC tissue-derived organoid of papillary carcinoma which had been continuously propagated for more than 6 months by using the organoid culture method [16]. It is important for us to study molecular pathogenesis and pathophysiology of uncommon pathological types $\mathrm{BC}$ as above. In this study, we describe the case of a 65-year-old Chinese woman with MPD and attempt to culture MPD-derived tumor cells using organoid culture method. To the best of our knowledge, this is the first report that presents the establishment of MPD patient-derived organoids.

\section{Materials and method}

\section{Patient and sample collection}

A 65-year-old Chinese woman was admitted with nipple change and mass in her left breast. The patient complained that molting of left nipple with small amount of white exudation for 7 months and rupture of left nipple 1 day ago. Broad bean sized mass was found in her left breast near the nipple 5 months ago. The patient had no family history. Physical examination revealed a hard mass under the nipple and areola, with unclear borders and irregular shapes. Rupture of the left nipple was $0.3 \times 0.3 \mathrm{~cm}$ with local blood exudation (Fig. 1b). The contralateral breast was normal and no axillary lymph nodes were palpable. The magnetic resonance imaging demonstrated that the mass in the left areola area with sunken skin, BI-RADS level 5 (Fig. 1a). The other examinations were negative and no distant metastases. The patient underwent modified radical mastectomy.

The postoperative gross pathologic inspection showed mammary glands and attached skin. Nipple was inverted, gray and tough swelling could be seen under the skin (Fig. 1c). Postoperative pathology results reported that both the nipple area and the mass behind the nipple were adenocarcinoma (Figs. 2 and 4). No axillary lymph node metastasis $(0 / 24)$. The immunohistochemistry pathology further confirmed the high expression of estrogen receptor (ER) and progesterone receptor (PR) (Fig. 4). This patient is currently receiving aromatase inhibitor Letrozole. At 1-year follow-up, the patient was free of disease.

The tumor sample was obtained from the MPD patient at the time of surgery. The $\mathrm{BC}$ tissue was treated as we had described before [16]. The study was conducted at The Second Hospital of Dalian Medical University (Dalian, China). The research protocol was approved and recorded by the Ethics Committee of The Second Hospital of Dalian Medical University. All procedures are carried out in accordance with the Helsinki Declaration.

\section{Organoids and tumor cells culture}

The tumor tissue was treated as we had described before [16]. The nipple tissue and tumor tissue was cut into pieces separately, washed with AdDF +++ (Advanced 

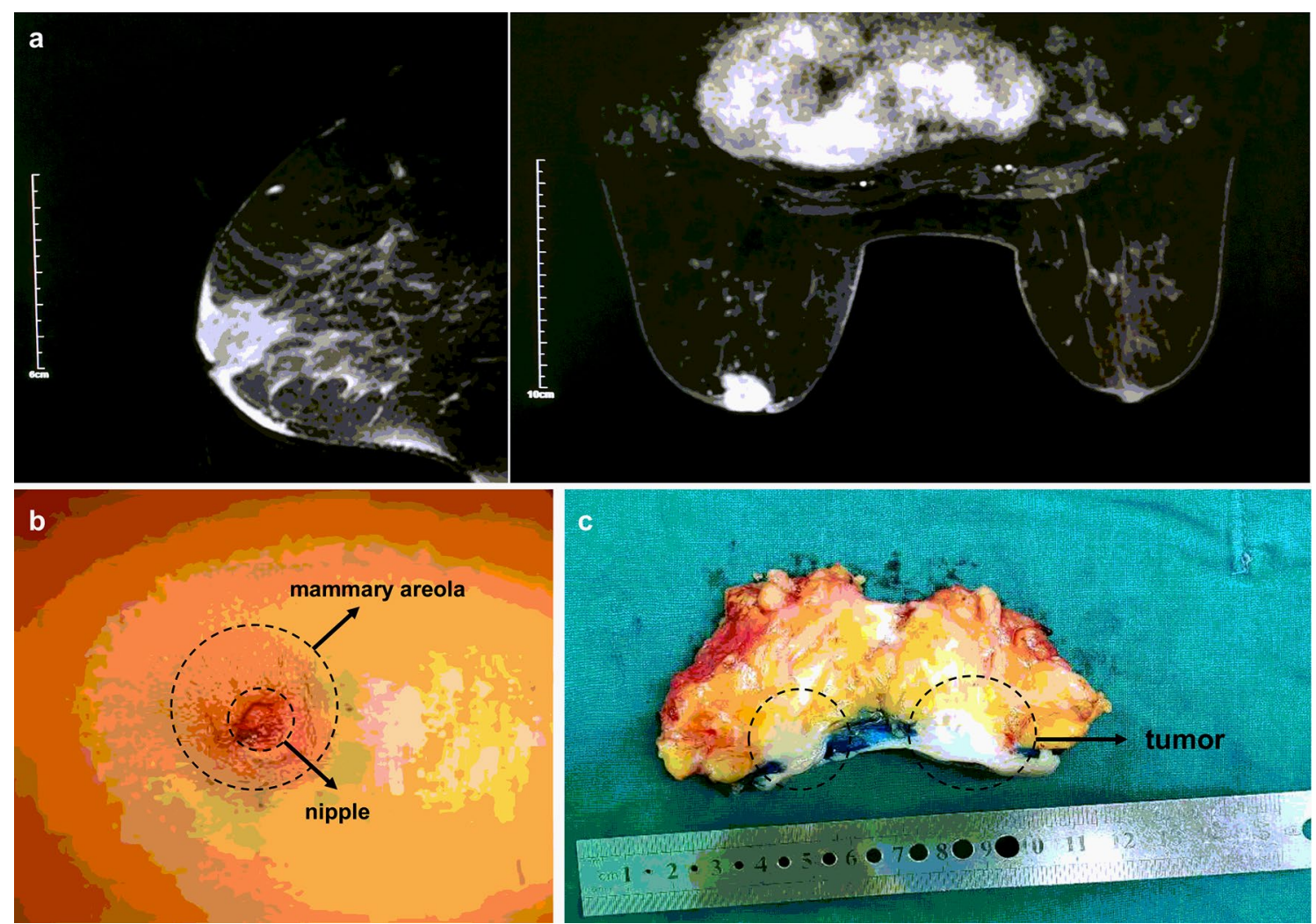

Fig. 1 MRI scan, visual examination and surgical specimen of mammary Paget's disease of right breast in a 65-year-old Chinese woman. a Chest MRI showed nodules in the left areola area with sunken skin. $\mathbf{b}$ Rupture of the left nipple was $0.3 \times 0.3 \mathrm{~cm}$ with local blood exudation. $\mathbf{c}$ The gross pathologic inspection showed mammary glands and attached skin. MRI: Magnetic resonance imaging

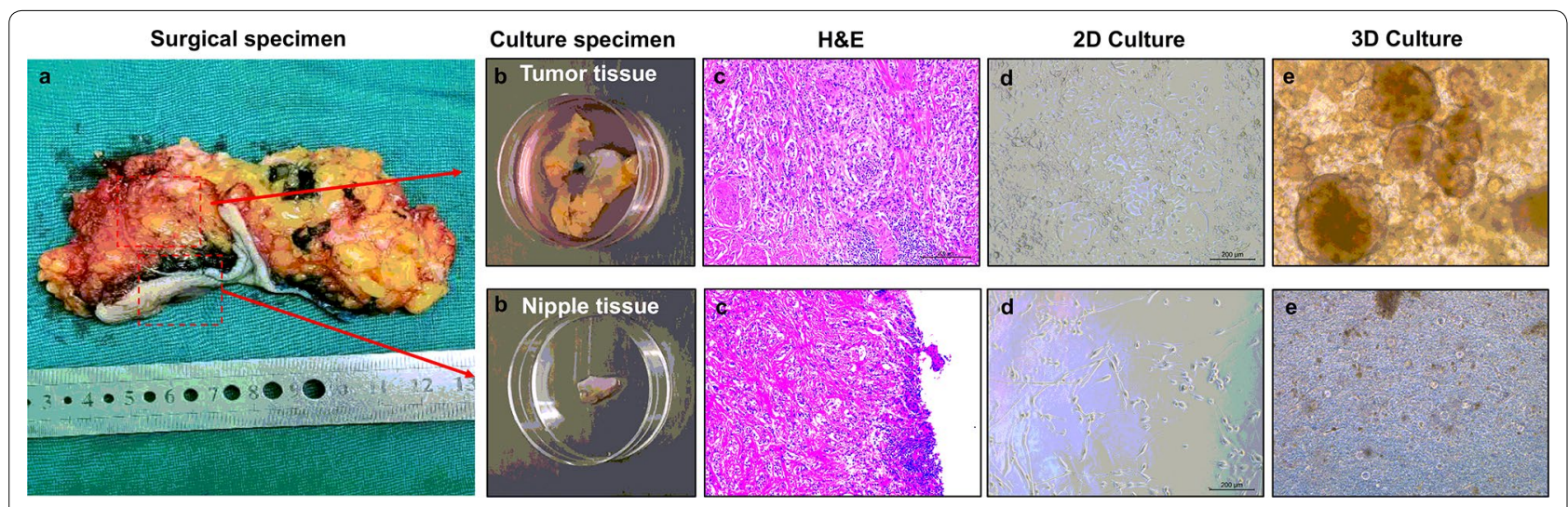

Fig. 2 Representative images of organoids cultured from a mammary Paget's disease patient. a Surgical specimen of mammary Paget's disease. b Cut an appropriate size of nipple tumor tissue and breast tumor tissue behind the nipple for culture and store respectively. c Postoperative pathology results reported that both the nipple area and the mass behind the nipple were adenocarcinoma. $\mathbf{d}$, e Culture tumor cells and organoids using nipple tumor tissue and breast tumor tissue under 2D and 3D cultivating mode separately

DMEM/F12 (Sigma, Saint Louis, MO, USA) containing $1 \times$ Glutamax (Invitrogen, Carlsbad, CA, USA), $10 \mathrm{mM}$ HEPES (Invitrogen, Carlsbad, CA, USA) and $100 \mathrm{U} /$ $\mathrm{mL} / 100 \mathrm{mg} / \mathrm{mL}$ Penicillin/Streptomycin (Sigma, Saint
Louis, MO, USA)) and digested in $3 \mathrm{~mL}$ medium [16] containing $1 \mathrm{mg} / \mathrm{mL}$ Collagenase (Sigma, Saint Louis, MO, USA) at $37^{\circ} \mathrm{C}$ for appropriate time. The digested tissue was collected in $3 \mathrm{~mL} \mathrm{AdDF+++}$. If a red precipitate 
is formed, red blood cells are lysed in $1 \mathrm{~mL}$ of red blood cell lysis buffer (Roche, Basel, Switzerland) for $3 \mathrm{~min}$ at room temperature and centrifuged at $1300 \mathrm{rpm}$ after adding $3 \mathrm{~mL}$ of AdDF +++ .

3D organoid culture model: The pellet was suspended in cold Cultrex growth factor reduced BME type 2 (Trevigen, Gaithersburg, MD, USA). $45 \mu \mathrm{L}$ of BME-cell suspension droplets were seeded in a preheated 24-well suspension plate (Corning Incorporated, NY, USA) at $37{ }^{\circ} \mathrm{C}$ for $25 \mathrm{~min}$. After gelation was completed, it was overlaid with $450 \mu \mathrm{L}$ medium. Passaging of organoids was performed as we had described before [16].

2D tumor cell culture model: The pellet was counted and $4 \times 10^{4}$ of tumor cells were seeded on 24-well plates (Nest, Wuxi, Jiangsu, China) with $500 \mu \mathrm{L}$ medium. The medium was changed and passaged every 3 days: $500 \mu \mathrm{L}$ of TrypLE Express (Invitrogen, Carlsbad, CA, USA) was added to the tumor cells which incubated for $5 \mathrm{~min}$ at $37{ }^{\circ} \mathrm{C} .1 .5 \mathrm{~mL}$ AdDF +++ was added and centrifugated at $1000 \mathrm{rpm}$. The pellet was reseeded in the ratio (1:3) as described above.

\section{Immunohistochemistry}

To maintain the 3D structure of the primary culture tumor cells, BME-cells mixture was aspirated from the 24-well plates gently and completely, then it was embedded in HistoGel (Biocoat, Corning, NY, USA). All samples were fixed in $4 \%$ paraformaldehyde before embedded in paraffin. The slides were incubated overnight with primary antibody Rabbit anti-ER antibody (1:5, ab27595), anti-PR (1:150, ab63605), anti-HER2 (1:600, ab134182), and anti-Ki67 (1:150, ab16667) which were purchased from Abcam (Cambridge, MA, UK). The DAB kit was purchased from Zhongshan Goldenbridge Biotechnology Company (Beijing, China). All procedures were carried out according to the manufacturer's instructions.

\section{Genomic DNA analysis}

The organoids and the oral epithelial cells were used for extraction of genomic DNA respectively, and subjected to whole genome sequencing by sequencing company (Shihe Gene, Nanjing, China). The detailed methods are described in Additional file 1: Data S1.

\section{In situ senescence-associated acid beta galactosidase assay}

Cell senescence $\beta$-galactosidase staining kit was purchased from Beyotime (Shanghai, China). Organoids grown in 24-well suspension plate (Corning Incorporated, NY, USA) were fixed using $\beta$-galactosidase staining fixative for $15 \mathrm{~min}$ at room temperature. After that, the organoids were incubated with a dyeing working fluid at $37{ }^{\circ} \mathrm{C}$ overnight. All procedures were carried out according to the manufacturer's instructions. The percentage of senescent cells was calculated by the number of blue, $\beta$-galactosidase-positive cells out of at least 500 cells in different microscope fields, as already reported [17].

\section{Results \\ Establishing MPD patient-derived organoids}

In order to evaluate the feasibility of primary culture of MPD-derived tumor cells, we used organoid technology to culture these tumor cells from our patient. Due to the consistency of the time and space of the nipple tumor and the tumor behind the nipple areola, we thought their tumor cells were likely to have the same origin. We obtained BC surgical specimen from this patient who underwent the mastectomy (Fig. 1b and c). We used nipple tumor tissue and tumor tissue behind the nipple to culture tumor cells under 2D cultivating mode and organoids under 3D cultivating mode separately. However, we failed to culture nipple tissue-derived tumor cells under both 2D and 3D condition. We only observed fibroblasts and it was difficult to observe tumor cells under 2D condition (Fig. 2). The culture state of nipple tissue-derived tumor cells under 3D condition was poor in the process of culture and died gradually even after passage (Fig. 2). On the other hand, we successfully cultured the tumor tissue behind the nipple-derived tumor cells under both 2D and 3D condition (Figs. 2 and 3). The tumor cells grew rapidly under $2 \mathrm{D}$ condition and the cultivation process at 1 day, 4 days and 7 days was recorded. When they were cultured for 4 days, they began to grow fast and fill up the entire cultivation space quickly after 4 days of cultivation (Additional file 2: Figure S1). The organoids grew relatively slowly under $3 \mathrm{D}$ condition and we recorded the cultivation process of day $1,5,10$ and 15 . When they were cultured under 3D condition for 10 days, they began to fill up the entire cultivation space quickly. The number of organoid masses increased rapidly and the volume increased gradually (Additional file 2: Figure S1). We observed dense and solid organoids and they were cohesive. (Figs. 2 and 3). After the organoids were cultured for 15 days, it could be seen that the growth of the organoids was obviously slow down and there was no significant change in the number and size of organoids (Additional file 2: Figure S1). The organoids were cultured for longterm expansion without any change in spherical organoid morphology (Fig. 3). Besides, the spherical organoid morphology did not change when they underwent cryopreservation after resuscitation (Fig. 3). We successfully established the MPD patient-derived organoids which had been continuously propagated for more than 6 months. 

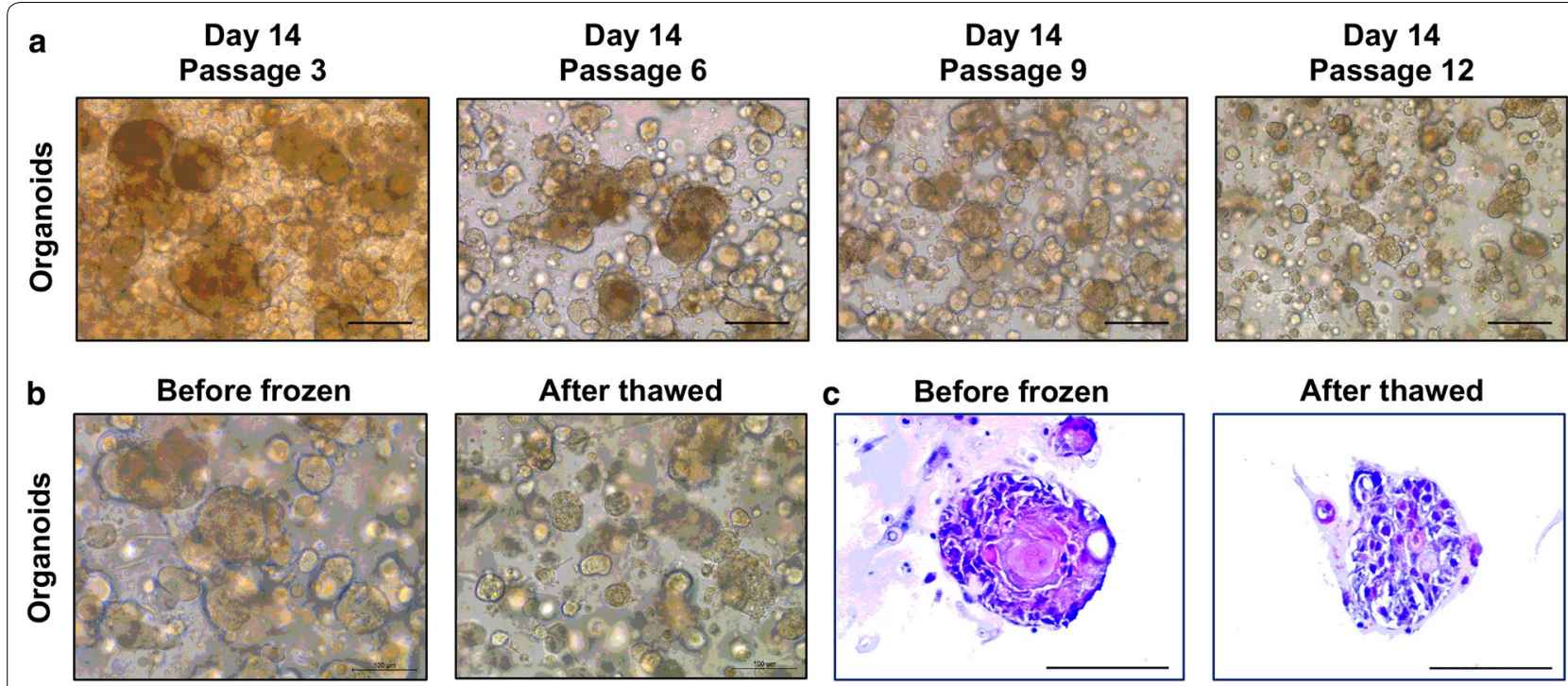

Fig. 3 Breast cancer organoids established from a mammary Paget's disease patient. a Representative images of long-term cultured breast cancer organoids without change in spherical organoid morphology. b, c The morphology of breast cancer organoids did not change before freezing and after thawing by Bright-field microscopy images and H\&E staining. Scale bar $=100 \mu \mathrm{m}$

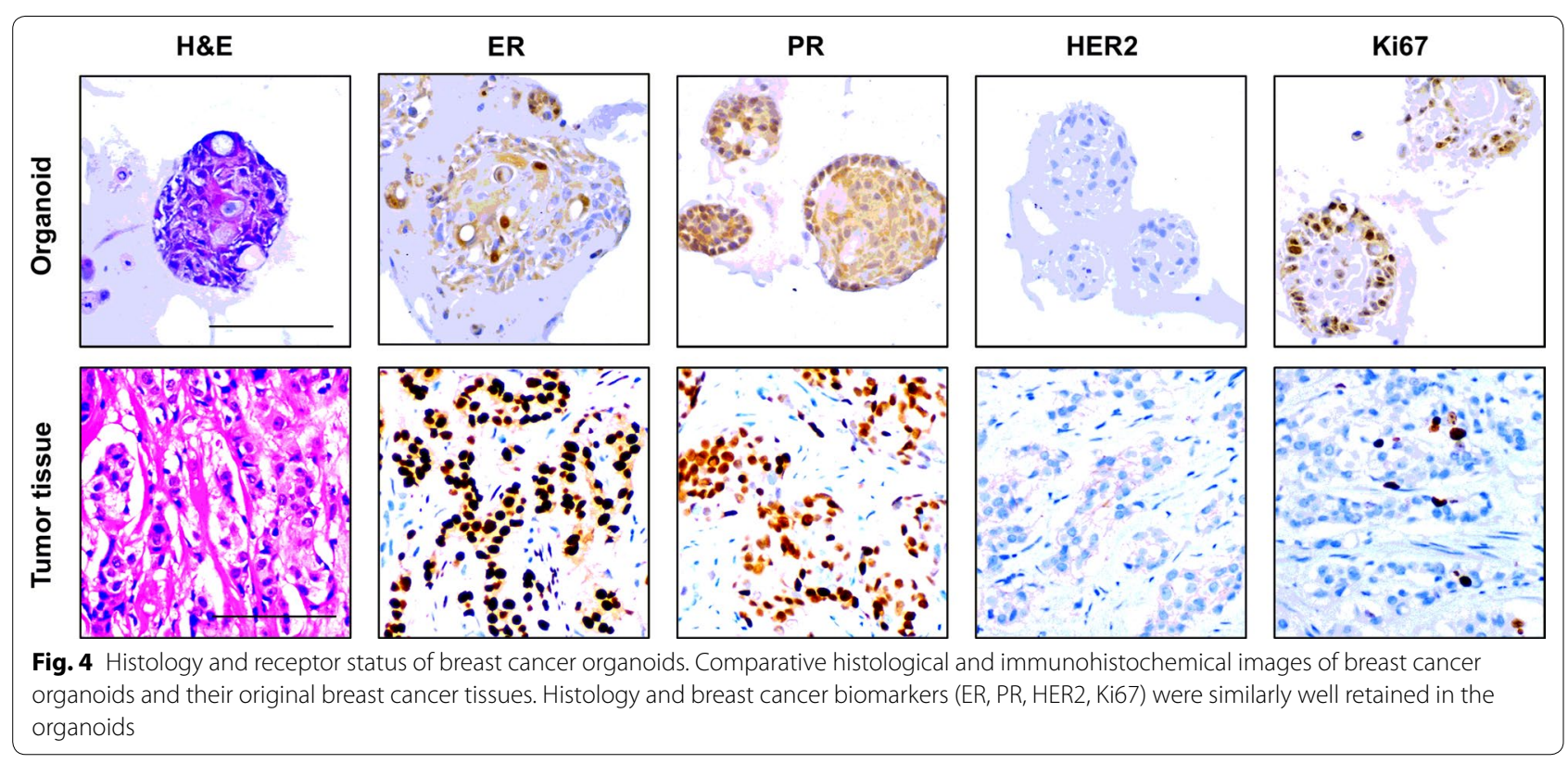

\section{MPD patient-derived organoids match the original histological characteristics}

We performed histopathological analysis of H\&E stained tissues and MPD patient-derived organoids sections, and confirmed that the phenotypes of tumor cells matched the histological characteristics of BC. Based on cellular and nuclear atypia, tumor cells clearly showed malignant characteristics. The cell size was also different and enlarged (Fig. 4). The spherical organoid morphology did not change when they underwent cryopreservation after resuscitation by H\&E staining (Fig. 3). Besides histological conservation, the organoids retained ER and PR positive expression of the $\mathrm{BC}$ biomarkers like primary $\mathrm{BC}$ and negative expression of human epidermal growth factor receptor 2 (HER2) (Fig. 4). The analysis of Immunohistochemistry also showed strongly positive staining of antigen Ki-67 (Fig. 4). Together, we found that MPD patient-derived organoids matched the primary tumor in 
histopathology and hormone receptor status, and could be a high-fidelity model.

\section{Genomic characterization of MPD patient-derived organoids}

Whole genome sequencing of oral epithelial cells and MPD patient-derived organoids was used to characterize genomes of MPD patient-derived organoids including copy number alterations, mutational signatures and somatic mutations. The organoids showed clean copy number signals and changes in copy number gains or losses were not obvious (Fig. 5a, b and Additional file 3: Figure S2). We analyzed base substitutions in organoids and plotted the total number of mutations per mutational signature. We found the organoids with more than 250 mutations in total as well as the relative contribution of individual signatures (Additional file 3: Figure S2). We identified significant contributions of major BC signatures in the organoids (Additional file 3: Figure S2), including Signature 1, Signature 3 and Signature 30. The point mutation type was dominated by $\mathrm{T}>\mathrm{G}$ and $\mathrm{C}>\mathrm{T}$ (Fig. 5c). Besides, the somatic mutations of the organoids were identified (Additional file 4: Data S2) and cancer mutant genes in the most relevant $\mathrm{BC}$ genes were also found, including ERBB4, HLA-DRB1, PDE4DIP, PTPN22 (Fig. 5d).

\section{Senescence is present in MPD patient-derived organoids}

To evaluate the presence of cellular senescence in breast cancer cells, we used MPD patient-derived organoids to perform in situ senescence-associated acid beta galactosidase assay. We selected the 3rd generation organoids, the 12th generation organoids and the resuscitated organoids for evaluation of the effect of passage and resuscitation on organoid senescence. Senescence organoid clusters were found in all three organoid cultures and larger organoid clumps were more stained. However, there were no significant statistical differences among them (Additional file 2: Figure S1). Therefore, senescence phenomenon existed in the process of organoids culture and there was no significant difference in the proportion of senescent organoids after organoid passage and resuscitation.

\section{Discussion}

Here we describe an effective platform for ex vivo expansion of BC tumor cells derived from a MPD patient. To our knowledge, this is the first report that presents the establishment of MPD patient-derived organoids. We proved the consistency of the morphological and histological features between the organoids and its original $\mathrm{BC}$ tissues by performing $\mathrm{H} \& \mathrm{E}$ staining and immunohistochemistry analysis. We found novel genomic characteristics of MPD patient-derived organoids including copy number alterations, mutational signatures and somatic mutations by whole genome sequencing. This platform could be used to explore clinicopathological and genomic characteristics of these patients.

At present, the precise origin of mammary Paget's cells has been debated for a long time. It has been proposed that they arise within the epidermis (intraepidermal theory) or from underlying breast carcinomas (epidermotropic theory) [6]. In this study, we tried to use nipple tumor tissue and tumor tissue behind the nipple to culture organoids. We failed to culture nipple tumor
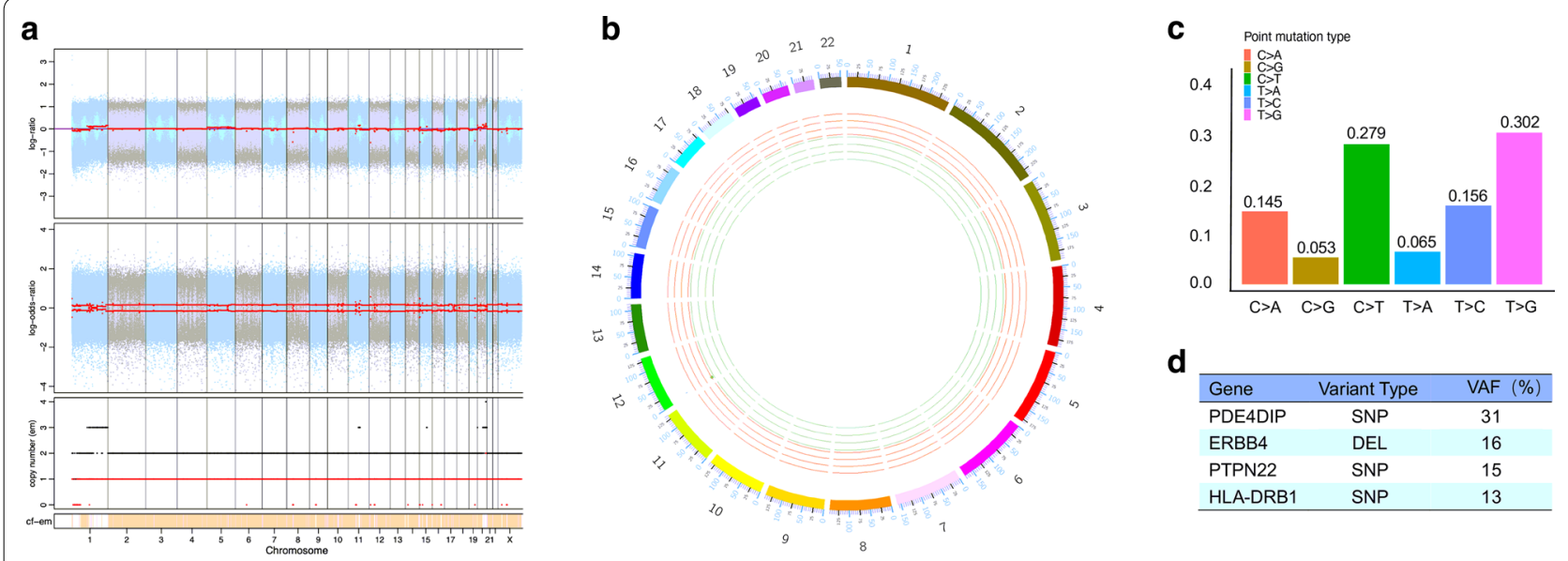

Fig. 5 Genomic Characterization of the organoids derived from a mammary Paget's disease patient. a, b Scatterplot and circos plot illustrating genome copy number alterations of the organoids which showed clean copy number signals and changes in copy number gains or losses were not obvious. c Bar graph showing the relative contributions of point mutation types underlying mutational signatures for the organoids. d Somatic mutations found in breast cancer genes of the organoids 
tissue-derived tumor cells, and too few nipple-derived tumor cells were the cause of cultivation failure. At present, no previous studies had reported primary culture of nipple tissue-derived tumor cells successfully and the proper culture conditions were not known. However, we successfully established $\mathrm{BC}$ organoids from tumor tissue near the nipple which had been continuously propagated for more than 6 months. We found that its unique morphological characteristics and the bright-field morphology of MPD patient-derived organoids were different from that of previously reported breast cancer patients $[13,16]$. We observed dense and solid organoids and they were cohesive. The organoids were cultured for longterm expansion without any change in spherical organoid morphology. The spherical organoid morphology did not change when they underwent cryopreservation after resuscitation. We thought this was a breakthrough in the field of primary cell culture of MPD.

Recent studies [18] found that the molecular subtype of the associated BC is usually similar to that of MPD. The HER2-enriched subtype is the most frequently occurring molecular subtype in MPD, followed by the luminal subtype. In this study, we successfully established $\mathrm{BC}$ organoids from a Luminal A subtype BC patient with MPD. We performed histopathological analysis of H\&E stained tissues and MPD-derived organoids sections, and confirmed that the organoids matched the histological characteristics of MPD by H\&E staining. Besides histological conservation, the organoids retained ER and PR positive expression of the breast cancer biomarkers like primary BC. The analysis of immunohistochemistry also showed strongly positive staining of Ki67.

The full genomic mutational landscapes of MPD remain uncharacterized, and genomics-related research is still scarce. Zhang et al. [7] performed whole-exome sequencing on 41 pairs of PD tumor and normal skin samples to reveal the molecular landscapes of PD. They found that MPD and EMPD were shown to have similar genomic aberrations, especially in genes involved in chromatin remodeling processes, such as $K M T 2 C$ and $A R I D 2$. Further, MPD and underlying breast ductal carcinomas are likely independent oncogenic events. KMT2C is likely an early oncogenic driver for PD. Gatalica et al. [8] investigated the molecular differences between MPD and EMPD. They found PIK3CA and TP53 mutations were most common in MPD. Although rare, several other genomic alterations were also detected in MPD, including CHEK2, CDK12, MLLT6 and MDM2. Copy number alterations affect a larger fraction of the cancer genome than any other type of genetic alterations [6]. Recent research found $\mathrm{BC}$ organoids recapitulated the original copy number alterations patterns of cancer genes and showed signal amplitude similarly to genome-wide copy number alterations specific BC genes. Copy number alterations were retained across several tumor-organoid pairs include CDKN2A, ERBB2, NF1, and SNX31 [13]. We employed whole genome sequencing to characterize genomes of MPD-derived organoids including copy number alterations, mutational signatures and somatic mutations. We found that the organoids showed clean copy number signals and changes in copy number gains or losses were not obvious.

We analyzed base substitutions in organoids and plotted the total number of mutations per mutational signature. We found the organoids with more than 250 mutations in total as well as the relative contribution of individual signatures. Diverse mutational processes result in distinct mutational signatures, at least 12 of which are found in $B C[19,20]$. We identified significant contributions of major $\mathrm{BC}$ signatures in the organoids, including Signature 1, Signature 3 and Signature 30. Signature 1 $[19,20]$ is the result of an endogenous mutational process initiated by spontaneous deamination of 5-methylcytosine. It is associated with small numbers of small insertions and deletions in most tissue types which correlates with age of cancer diagnosis. Signature $3[19,20]$ is associated with failure of DNA double-strand breakrepair by homologous recombination. It has relationship with elevated numbers of large (longer than $3 \mathrm{bp}$ ) insertions and deletions with overlapping microhomology at breakpoint junctions. It also associated with germline and somatic BRCA1 and BRCA2 mutations in breast. Signature $30[19,20]$ has been observed in a small subset of breast cancers and the aetiology of Signature 30 remains unknown.

The point mutation type was dominated by $\mathrm{T}>\mathrm{G}$ and $\mathrm{C}>\mathrm{T}$ and similar to previously described mutational signatures for PD [7]. The somatic mutations of the organoids were identified and cancer genes in the most relevant $\mathrm{BC}$ genes were also found, including $E R B B 4$, HLA-DRB1, PDE4DIP, PTPN22.

It is similar to the previously described research that $\mathrm{BC}$ organoids displayed mutations in many of the most relevant $\mathrm{BC}$ genes [13].

Generally, senescent cells are observed as human beings age. They are also observed in normal organ development and pathological conditions related to aging $[21,22]$. Most of the research on senescent cells has been conducted in primary isolated normal cells, and in vitro studies have induced cellular senescence mainly through DNA stimulation or subculture of fibroblasts [23]. Recently, cellular senescence has been observed in tumor tissues and may be involved in cancer progression [24, 25]. These senescent cells are thought to originate from tumor cells and are called senescent tumor cells [26]. Senescent tumor cells are observed in various carcinomas 
$[27,28]$ but it was rarely reported in breast cancer. In some carcinomas, the distribution of senescent tumor cells is not even, and it is observed in specific tumor tissue locations. Senescent tumor cells do not exist in the center of the mass where hypoxic damage usually occurs, but rather in the marginal region of the tumor [28]. Furthermore, they are present in large numbers in the metastatic lymph nodes and lymphatic vessels [28]. These data demonstrate that senescent tumor cells are involved in cancer progression. To evaluate the presence of cellular senescence in primary cultured breast cancer cells, we used MPD patient-derived organoids to perform in situ senescence-associated acid beta galactosidase assay. We selected the 3rd generation organoids, the 12th generation organoids and the resuscitated organoids for evaluation of the effect of passage and resuscitation on organoid senescence. Senescence organoid clusters were found in all three organoid cultures and larger organoid clumps were more stained. However, there were no significant statistical differences among them. Therefore, senescence phenomenon existed in the process of organoids culture and there was no significant difference in the proportion of senescent organoids after organoid passage and resuscitation. The major limitation of the current study is due to the analysis of only one patient. The effect of senescent tumor cells on tumors and tumour progression remains controversial which is still to be further studied. In the future, we plan to build a larger-scale organoid model of MPD patients which will contribute to elucidation of pathophysiological mechanisms of MPD. It accelerates both basic and translational research and promotes better prognosis for patients with MPE.

\section{Conclusion}

We successfully established a novel $\mathrm{BC}$ organoid model from a MPD patient which could be used to explore clinicopathological and genomic characteristics of these patients.

\section{Supplementary information}

Supplementary information accompanies this paper at https://doi. org/10.1186/s12935-020-01459-6.

Additional file 1: Data S1. The experimental procedure of whole genome sequencing.

Additional file 2: Figure S1. Representative images of successful 2D and $3 \mathrm{D}$ growth process and senescence evaluation of the organoids derived from a mammary Paget's disease patient. a. The tumor cells grew rapidly under 2D condition and the cultivation process at 1 day, 4 days and 7 days was recorded. They began to fill up the entire cultivation space quickly after 4 days of cultivation. Scale bar $=200 \mu \mathrm{m}$. b. The organoids grew relatively slowly under 3D condition and we recorded the cultivation process of day 1,5,10 and 15. When they were cultured under 3D condition for 10 days, they began to fill up the entire cultivation space quickly. Scale bar $=200 \mu \mathrm{m}$. c. We found the phenomenon of senescence during organoid culture. Senescence phenomenon existed in the process of organoids culture and there was no significant difference in the proportion of senescent organoids after organoid passage and resuscitation. Scale bar $=100 \mu \mathrm{m}$. The graph shows the mean percentage of the senescent 3 rd generation organoids, the 12 th generation organoids and the resuscitated organoids. Mean \pm SD of results from 3 independent field of microscope is shown.

Additional file 3: Figure S2. Genomic Characterization of the organoids derived from a mammary Paget's disease patient. a. Heatmap showing copy number alterations in coding DNA sequences of breast cancer genes. b. Stacked bar graph showing the total mutation load per mutational signature of the organoids. Typical breast cancer mutational signatures (bold) were present and conserved.

Additional file 4: Data S2. The somatic mutations of the organoids.

\section{Abbreviations}

PD: Paget's disease; MPD: Mammary Paget's disease; EMPD: Extramammary Paget's disease; BC: Breast cancer; DCIS: Intraductal carcinoma; IDC: Infiltrating duct carcinoma; 3D: Three-dimensional; 2D: Two-dimensional; ER: Estrogen receptor; PR: Progesterone receptor; HER2: Human epidermal growth factor receptor 2; Ki67: Antigen Ki-67.

\section{Acknowledgements}

We thank the mammary Paget's disease patient enrolled in this study, and is indebted to Professor Zhenhai Ma for the clinical data.

\section{Authors' contributions}

$\mathrm{BP}, \mathrm{DZ}, \mathrm{YL}, \mathrm{NL}, \mathrm{CS}$ and NL performed experiments and analysis and prepared the manuscript. $B P, D Z, Y L, X L, Z Z$ and $M L$ conceived and designed the experiments. All authors read and approved the final manuscript.

\section{Funding}

This work was supported by the National Natural Science Foundation of China (No. 81673762 to Zuowei Zhao, No. 81872156 to Man Li), Provincial Foundation of Liaoning (No. LR2017012 to Zuowei Zhao, No. 2019-BS-072 to Xuelu Li), and Innovation Foundation of Dalian (No. 2018J11 CY026 to Zuowei Zhao).

\section{Availability of data and materials}

All data during this research are included in this published article.

\section{Ethics approval and consent to participate}

Tumor specimens were obtained with the approval of the ethics committee of The Second Hospital of Dalian Medical University. Written informed consent was obtained from the patient.

\section{Consent for publication}

Not applicable.

\section{Competing interests}

The authors declare that they have no competing interests.

\section{Author details}

${ }^{1}$ Department of Oncology \& Department of Breast Surgery, The Second Hospital of Dalian Medical University, Dalian 116023, China. ${ }^{2}$ Department of Foreign Language, Dalian Medical University, Dalian 116000, China.

Received: 20 May 2020 Accepted: 25 July 2020

Published online: 03 August 2020

\section{References}

1. Gaurav A, Gupta V, Koul R, et al. Practical consensus recommendations for Paget's disease in breast cancer. South Asian J Cancer. 2018;7(2):83-6.

2. Apalla Z, Errichetti E, Kyrgidis A, et al. Dermoscopic features of mammary Paget's disease: a retrospective case-control study by the International Dermoscopy Society. J Eur Acad Dermatol Venereol. 2019;33(10):1892-8. 
3. Waldman RA, Finch J, Grant-Kels JM, Stevenson C, Whitaker-Worth D. Skin diseases of the breast and nipple: benign and malignant tumors. J Am Acad Dermatol. 2019;80(6):1467-81.

4. Zhou H, Lu K, Zheng L, et al. Prognostic significance of mammary Paget's disease in Chinese women: a 10-year, population-based, matched cohort study. Onco Targets Ther. 2018;11:8319-26.

5. Zhao Y, Sun HF, Chen MT, et al. Clinicopathological characteristics and survival outcomes in Paget disease: a SEER population-based study. Cancer Med. 2018:7(6):2307-18.

6. Lopes Filho LL, Lopes IM, Lopes LR, et al. Mammary and extramammary Paget's disease. An Bras Dermatol. 2015;90(2):225-31.

7. Zhang G, Zhou S, Zhong W, et al. Whole-exome sequencing reveals frequent mutations in chromatin remodeling genes in mammary and extramammary Paget's diseases. J Invest Dermatol. 2019;139(4):789-95.

8. Gatalica Z, Vranic S, Krušlin B, et al. Comparison of the biomarkers for targeted therapies in primary extra-mammary and mammary Paget's disease. Cancer Med. 2020;9(4):1441-50.

9. Calandrini C, Schutgens F, Oka R, et al. An organoid biobank for childhood kidney cancers that captures disease and tissue heterogeneity. Nat Commun. 2020:11(1):1310.

10. Ooft SN, Weeber F, Dijkstra KK, et al. Patient-derived organoids can predict response to chemotherapy in metastatic colorectal cancer patients. Sci Transl Med. 2019;11(513):eaay2574.

11. Driehuis $E$, van Hoeck $A$, Moore $K$, et al. Pancreatic cancer organoids recapitulate disease and allow personalized drug screening. Proc Natl Acad Sci USA. 2019;116(52):26580-90.

12. Kim M, Mun H, Sung CO, et al. Patient-derived lung cancer organoids as in vitro cancer models for therapeutic screening. Nat Commun. 2019:10(1):3991.

13. Sachs $N$, de Ligt J, Kopper $\mathrm{O}$, et al. A living biobank of breast cancer organoids captures disease heterogeneity. Cell. 2018;172(1-2):373-86.

14. Li X, Zhu D, Li N, et al. Characterization of ascites-derived tumor cells from an endometrial cancer patient. Cancer Sci. 2017;108(12):2352-7.

15. Li X, Zhang Y, Ding J, et al. Clinical significance of detecting CSF-derived tumor cells in breast cancer patients with leptomeningeal metastasis. Oncotarget. 2018;9(2):2705-14.

16. Li $X$, Pan B, Song $X$, et al. Breast cancer organoids from a patient with giant papillary carcinoma as a high-fidelity model. Cancer Cell Int. 2020;20:86.
17. Alessio N, Squillaro T, Di Bernardo G, et al. Increase of circulating IGFBP-4 following genotoxic stress and its implication for senescence. Elife. 2020:9:e54523.

18. Arafah M, Arain SA, Raddaoui EMS, et al. Molecular subtyping of mammary Paget's disease using immunohistochemistry. Saudi Med J. 2019;40(5):440-6

19. Alexandrov LB, Nik-Zainal S, Wedge DC, et al. Signatures of mutational processes in human cancer. Nature. 2013;500(7463):415-21.

20. Nik-Zainal S, Davies H, Staaf J, et al. Landscape of somatic mutations in 560 breast cancer whole-genome sequences. Nature. 2016;534(7605):47-54.

21. Storer M, Mas A, Robert-Moreno A, et al. Senescence is a developmental mechanism that contributes to embryonic growth and patterning. Cell. 2013:155(5):1119-30.

22. Muñoz-Espín D, Cañamero M, Maraver A, et al. Programmed cell senescence during mammalian embryonic development. Cell. 2013;155(5):1104-18.

23. Kuilman T, Michaloglou C, Mooi WJ, Peeper DS. The essence of senescence. Genes Dev. 2010;24(22):2463-79.

24. Childs BG, Durik M, Baker DJ, van Deursen JM. Cellular senescence in aging and age-related disease: from mechanisms to therapy. Nat Med. 2015;21(12):1424-35

25. Althubiti M, Lezina L, Carrera S, et al. Characterization of novel markers of senescence and their prognostic potential in cancer. Cell Death Dis. 2014:5(11):e1528.

26. Kim YH, Choi YW, Lee J, Soh EY, Kim JH, Park TJ. Senescent tumor cells lead the collective invasion in thyroid cancer. Nat Commun. 2017:8:15208.

27. Haugstetter AM, Loddenkemper C, Lenze D, et al. Cellular senescence predicts treatment outcome in metastasised colorectal cancer. $\mathrm{Br} J$ Cancer. 2010;103(4):505-9.

28. Kim YH, Choi YW, Han JH, et al. TSH signaling overcomes B-RafV600Einduced senescence in papillary thyroid carcinogenesis through regulation of DUSP6. Neoplasia. 2014;16(12):1107-20.

\section{Publisher's Note}

Springer Nature remains neutral with regard to jurisdictional claims in published maps and institutional affiliations.
Ready to submit your research? Choose BMC and benefit from:

- fast, convenient online submission

- thorough peer review by experienced researchers in your field

- rapid publication on acceptance

- support for research data, including large and complex data types

- gold Open Access which fosters wider collaboration and increased citations

- maximum visibility for your research: over 100M website views per year

At $\mathrm{BMC}$, research is always in progress.

Learn more biomedcentral.com/submissions 